\title{
Aplicação de Bandagem Elástica Funcional nos músculos expiratórios de portadores de Doença Pulmonar Obstrutiva Crônica
}

\author{
Functional Elastic Bandage application in expiratory muscles of \\ patients with Chronic Obstructive Pulmonary Disease
}

Natana Marques da Silva', Cássia da Luz Goulart', Daniela Angri', Lisiane Lisboa Carvalho', Ana Cristina Sudbrack', Andréa Lúcia Gonçalves da Silva'

"Universidade de Santa Cruz do Sul (UNISC), Santa Cruz do Sul, RS, Brasil.

Recebido em: julho 2015 / Aceito em: agosto 2015

andreag@unisc.br

\section{RESUMO}

Objetivo: verificar o efeito da Bandagem Elástica Funcional (BEF) aplicada nos músculos expiratórios (reto abdominal e oblíquo externo) sobre o pico de fluxo expiratório de portadores de Doença Pulmonar Obstrutiva Crônica (DPOC). Método: estudo quase-experimental, amostragem de conveniência, avaliou dois grupos: grupo bandagem elástica funcional (GBEF) e grupo controle (GC) pelas variáveis clínicas: nível de dispneia pela escala Medical Research Council; pico de fluxo expiratório (PFE) em três momentos (antes, imediatamente após e 5 dias após a aplicação da BEF). Resultados: o PFE, após aplicação da BEF, não apresentou diferença significativa na comparação com o GC. No grupo GBEF, logo após a aplicação da BEF, houve melhora do PFE, porém este decai na $3^{a}$ medida. Observou-se melhora clínica da dispneia nos diferentes momentos de análise do pico de fluxo expiratório. Considerações finais: a aplicação da BEF nos músculos expiratórios reto abdominal e oblíquo externo exerceu efeito positivo sobre o PFE dos portadores de DPOC com idade menos avançada, menor grau de obstrução de vias aéreas, eutróficos e em estadiamento avançado da doença.

Palavras-chave: DPOC; Fisioterapia; Músculos, Expiração.

\section{ABSTRACT}

Objective: assess the functional elastic bandage (FEB) effect on expiratory muscles (rectus abdominis and external oblique) on the expiratory peak flow (EPF) in patients with Chronic Obstructive Pulmonary Disease (COPD). Method: quasi-experimental design study with convenience sampling, the study evaluated two groups: functional elastic bandage group (FEBG) and control group (CG). Clinical variables assessed: level of dyspnea by Medical Research Council-scale; expiratory peak flow at three times (before, immediately after and 5 days after application of FEB). Results: The EPF after the FEB did not differ significantly between the FEBG and CG. In FEBG immediately after application of the FEB had improved EPF, but this declines in 3rd measure. There was clinical improvement in dyspnea at different times of analysis of EPF. Closing remarks: the application of FEB on rectus abdominis and external oblique had a positive effect on the EPF in younger age patients with COPD, with lower degree of airway obstruction, good $B M I$ and at an advanced stage of the disease.

Keywords: COPD; Physiotherapy; Muscles; Expiration.

\section{INTRODUÇÃO}

A Fisioterapia, hoje em dia, vem abordando várias técnicas para aperfeiçoar a expiração em pacientes com Doença Pulmonar Obstrutiva Crônica (DPOC), a fim de Ihes fornecer uma melhora na sua qualidade de vida. ${ }^{1} \mathrm{~A}$ DPOC pode ser distinguida como uma doença comum prevenível e tratável, caracterizada por obstrução nas vias aéreas, que é geralmente a resposta inflamatória do pulmão à partículas nocivas de gases. A limitação do fluxo aéreo é característica crônica da DPOC, causada 
por uma mistura de doença de pequenas vias aéreas (bronquite obstrutiva) e destruição do parênquima pulmonar (enfisema). Exacerbações e comorbidades contribuem para a gravidade global dos portadores de DPOC. Em função destas alterações, esses pacientes ficam com dificuldade de realizar uma expiração completa, devido uma diminuição do componente elástico nos pulmões, decorrente a uma ruptura das ligações entre os alvéolos. ${ }^{2}$ Assim, certa quantidade de dióxido de carbono $\left(\mathrm{CO}_{2}\right)$ proporcional à destruição dos pulmões que não foi expirada fica retido no interstício pulmonar.

Nesse sentido, os doentes com DPOC podem desenvolver hipercapnia crônica, em dado momento da sua história, correspondendo ao aparecimento de uma fase de maior gravidade clínica da doença. Os mecanismos fisiopatológicos que levam a uma hipercapnia ainda são obscuros, apesar da retenção de $\mathrm{CO}_{2}$ estar na dependência da gravidade da obstrução das vias aéreas. Ainda, há outros fatores relacionados como condicionadores de retenção de $\mathrm{CO}_{2}$, sendo eles a alteração na ventilação-perfusão, a perturbação do comando ventilatório, a fraqueza dos músculos respiratórios, o próprio padrão respiratório e a hiperinsuflação pulmonar. ${ }^{3}$

A reabilitação pulmonar (RP) é designada para reduzir os sintomas em portadores desta doença, aperfeiçoar o estado funcional, aumentar a adesão ao tratamento e reduzir os custos com a saúde. O tratamento por meio da RP melhora a dispneia, a capacidade para o exercício e o estado de saúde de forma mais eficaz que outras terapias, por estabilizar ou reverter as manifestações sistêmicas. Mesmo assim, estratégias para melhorar o funcionamento dos músculos são necessárias para reduzir a limitação funcional, a hiperinsuflação pulmonar e retenção de $\mathrm{CO}_{2}$, pois a reabilitação pulmonar não melhora diretamente a função pulmonar. ${ }^{4}$

Uma série de novas técnicas e métodos de reabilitação funcional vem surgindo na Fisioterapia, e sendo implementados nos programas de RP. Um desses novos recursos é a bandagem elástica funcional, cuja aplicabilidade apresenta-se cada vez mais diversificada dentro da área de atuação fisioterapêutica. As bandagens elásticas funcionais foram criadas no Japão pelo Dr. Kenzo Kase, sob a denominação de Kinesio Taping, e são consideradas pelos fisioterapeutas um método de auxílio na reabilitação das alterações do sistema neuromusculoesquelético; no entanto, por tratar-se de um método de intervenção recente, ainda carece de informações através de pesquisas clínicas bem delineadas. ${ }^{5}$

Entende-se que as bandagens elásticas funcionais promovam a normalização do tônus muscular, ação esta que se deve à combinação de efeitos mecânicos e estimulantes. A ação fisiológica da bandagem elástica funcional está fundamentada no princípio da estimulação exteroceptiva cutânea, formando um arco neural (estímulo-resposta), bem como pelo efeito mecânico produzido pelo tensionamento direcional da pele através da força reativa. ${ }^{5}$ Este tensionamento é retransmitido aos tecidos subjacentes (gordura, fáscia, músculo), diminuindo ou aumentando a contratilidade dos músculos, gerando assim efeitos sobre o tecido muscular que será abordado.

Neste sentido, este estudo buscou verificar em que medida a aplicação da bandagem elástica funcio- nal, nos músculos expiratórios reto abdominal e oblíquo externo, exerce algum efeito sobre o fluxo expiratório dos portadores de DPOC em programa de reabilitação pulmonar. Nós hipotetizamos que a aplicação da bandagem elástica funcional melhora o pico de fluxo expiratório dos portadores de DPOC, ao longo dos dias de aplicação da banda elástica, diminuindo a sensação de dispneia.

\section{MÉTODO}

Estudo do tipo quase-experimental foi composto por amostragem de conveniência realizada junto aos portadores de DPOC participantes de um Programa de Reabilitação Pulmonar (RP) localizado no Hospital Santa Cruz, em Santa Cruz do Sul/RS. Foram incluídos no estudo os portadores de DPOC com diagnóstico clínico de doença confirmado mediante prova de função pulmonar, independente do estadiamento da doença, eutróficos e com sobrepeso, sem exacerbação da doença nos últimos 15 dias e que compareciam regularmente à RP. Foram excluídos os portadores de DPOC desnutridos (índice de IMC $<17 \mathrm{Kg} / \mathrm{m}^{2}$ ), com presença de algum tipo de dermatite na região abdominal, que se recusaram a fazer a depilação dos pelos no local da aplicação da bandagem elástica funcional, bem como sujeitos com dificuldade de oclusão oral e que apresentaram broncoespasmo mediante ausculta pulmonar.

Este estudo foi aprovado pelo Comitê de Ética em Pesquisa da Universidade de Santa Cruz do Sul sob protocolo $n^{\circ} 868.985$ e os participantes deste estudo participaram de livre e espontânea vontade mediante assinatura do Termo de Consentimento Livre e Esclarecido. Após inclusão dos sujeitos no estudo, os mesmos foram então divididos em dois grupos: grupo bandagem elástica funcional (GBEF) e grupo controle (GC). O GBEF foi constituído pelos portadores de DPOC que atenderam os critérios de inclusão do estudo, em que foi utilizada a BEF da marca Kinesiology ${ }^{\circledR}$, totalizando 3 rolos de 5 metros cada, nas cores bege e verde, as cores foram escolhidas aleatoriamente. Já o GC foi constituído pelos portadores de DPOC ingressantes em outro projeto de pesquisa dentro da RP e, portanto, não foram submetidos à aplicação da bandagem elástica funcional.

Além das variáveis clínicas, foram analisadas as seguintes variáveis: Índice de Massa Corporal (IMC), nível de dispneia através da escala Medical Research Council-MRC; pico de fluxo expiratório pelo medidor de fluxo expiratório Peak Flow Meter ${ }^{\circledR}$. No GBEF, foram realizados três encontros para aplicação da bandagem elástica funcional nos músculos abdominais expiratórios (reto abdominal e oblíquo externo), sendo realizado o teste de pico de fluxo expiratório (Figura 1).

Todos os participantes inicialmente realizaram o teste do pico de fluxo expiratório e analisados em percentual do predito. O teste é realizado com o paciente em sedestação; o medidor de fluxo expiratório possui um bocal onde o paciente faz uma expiração forçada a partir de uma inspiração com esforço respiratório máximo até atingir sua capacidade pulmonar total. ${ }^{6}$

No segundo momento deste $1^{\circ}$ encontro, somente no grupo GBEF foi aplicada a bandagem elástica fun- 


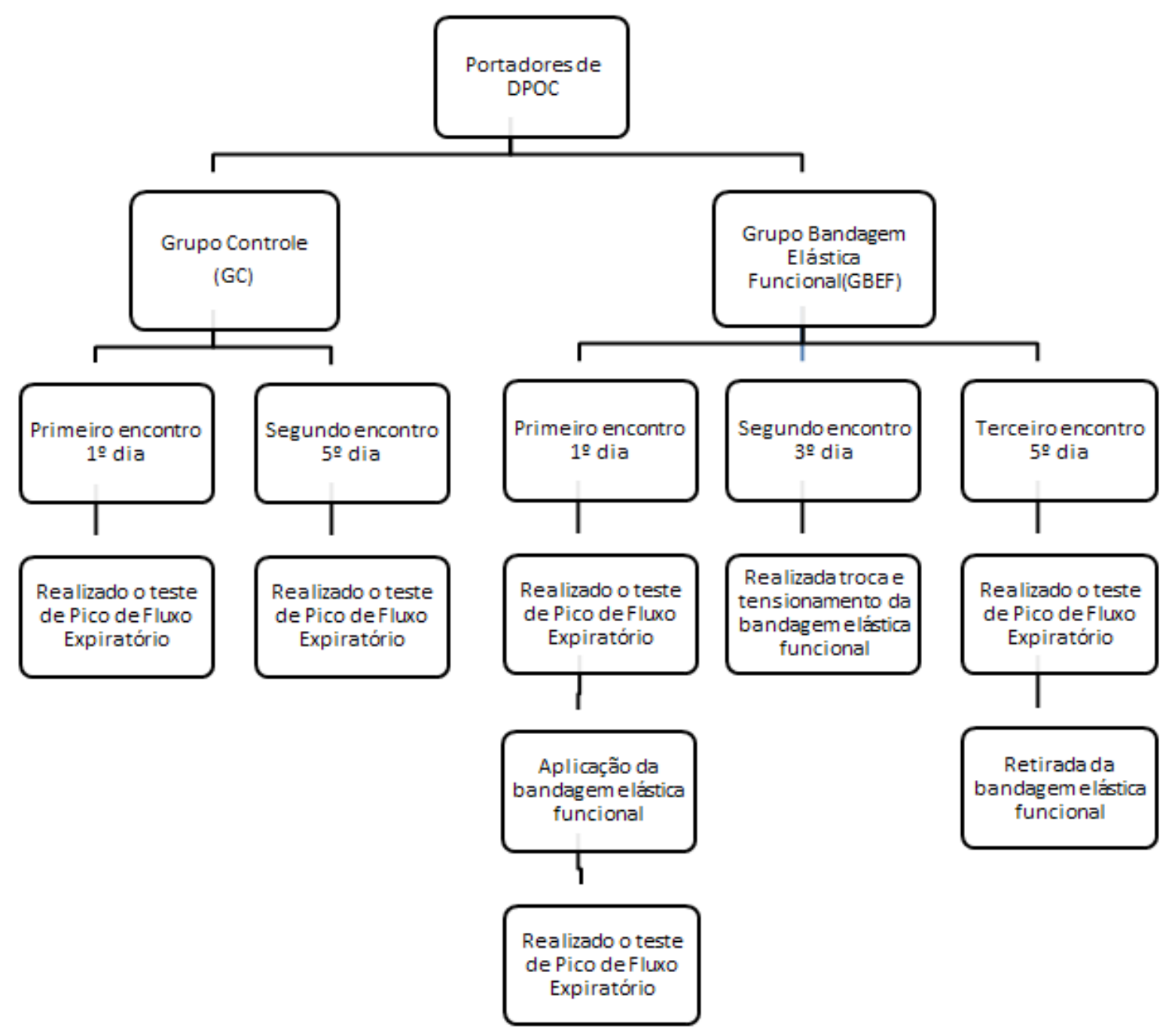

Figura 1 - Fluxograma das etapas da pesquisa.

cional, sobre os músculos reto abdominal e oblíquos externos dos pacientes que se encontravam na posição de decúbito dorsal. Os pacientes eram orientados a contrair a musculatura no momento em que a bandagem era aplicada e tensionada sobre a musculatura na direção da sua origem a sua inserção. ${ }^{7}$ Logo após a aplicação da bandagem elástica funcional, foi realizado novamente o teste de pico de fluxo expiratório.

O $2^{\circ}$ encontro deu-se 02 dias após aplicação das bandagens elásticas funcionais e neste momento, foram trocadas as bandagens e novamente tensionadas. Dois dias após, houve o $3^{\circ}$ encontro para retirada das bandagens e realização do teste de pico de fluxo expiratório. A tensão da bandagem elástica funcional aplicada neste estudo foi $>$ que $45 \%$ do seu tamanho original. ${ }^{8}$

No GC realizou-se apenas dois encontros, em que foi realizado o levantamento das variáveis clínicas e a medição do pico de fluxo expiratório. Estes encontros tiveram um intervalo de 04 dias entre a primeira e a segunda medida. Todos os resultados obtidos nesta pesquisa foram registrados em ficha específica. Para compor as variáveis analisadas deste estudo, foram coletados os dados referentes à função pulmonar, que se encontravam junto às avaliações clínicas contidas em um banco de dados computadorizado dos pacientes pertencente à RP. Ressalta-se que os participantes de ambos os grupos realizaram normalmente suas atividades no programa de Reabilitação Pulmonar durante as coletas deste estudo.

\section{Análise Estatística}

Para a análise dos dados foi utilizado o programa Statistical Package for Social Science (SPSS 20.0, EUA), sendo os mesmos descritos em média e desvio padrão. Para as variáveis numéricas utilizou-se teste $t$ student e nas categóricas, teste $x^{2}$ quadrado, a avaliação média do Peak Flow antes, imediatamente e após quatro dias, foi utilizado o teste de Variância (ANOVA) two-way seguindo de post hoc de Tukey, considerando um significativo de $\mathrm{p}<0,05$.

\section{RESULTADOS E DISCUSSÃO}

O estudo foi constituído por 18 portadores de DPOC, dos quais 10 eram do sexo masculino e 08 do sexo feminino, todos com história de tabagismo prévio ao estudo. As características clínicas estão descritas na (Tabela 1).

De um modo geral, observou-se uma frequência discretamente maior de portadores de DPOC do sexo masculino no grupo GC, o que corrobora com o que 
Tabela 1 - Características clínicas dos portadores de DPOC.

\begin{tabular}{lccc}
\hline Variáveis & $\begin{array}{c}\text { Grupo GC } \\
\text { Total }=\mathbf{6}\end{array}$ & $\begin{array}{c}\text { Grupo GBEF } \\
\text { Total }=11\end{array}$ \\
\hline Sexo masculino, $\mathrm{n}(\%)^{\mathrm{b}}$ & $4(66,7)$ & $6(54,5)$ & Valor de $\boldsymbol{p}^{*}$ \\
Idade $($ anos) & $63,83 \pm 9,66$ & $60,82 \pm 8,75$ & 0,25 \\
IMC $\left(\mathrm{Kg} / \mathrm{m}^{2}\right)^{\mathrm{a}}$ & $23,33 \pm 4,50$ & $25,18 \pm 5,10$ & 0,66 \\
Magreza, $\mathrm{n}(\%)^{\mathrm{b}}$ & $2(33,3)$ & $4(36,4)$ & 0,46 \\
Eutrófico, $\mathrm{n}(\%)^{\mathrm{b}}$ & $3(50,0)$ & $2(18,2)$ & 0,41 \\
Obesidade, $\mathrm{n}(\%)^{\mathrm{b}}$ & $1(16,7)$ & $5(45,5)$ & 0,10 \\
\hline
\end{tabular}

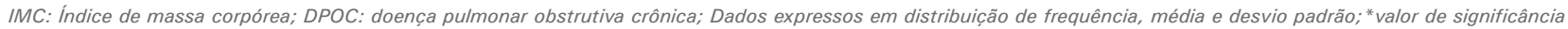
de $p$; ${ }^{a}$ :Teste $t$ de Student; ${ }^{b}$ :Teste $\times 2$ quadrado.

a literatura nos trás, pois o sexo masculino é o mais acometido pela DPOC, devido ao fato de que homens fumam mais do que mulheres ${ }^{9}$. A idade adulta avançada também é outra característica dos portadores de DPOC, pois os sintomas respiratórios variam de acordo com a idade, ou seja, geralmente detectada após os 40 anos de idade onde a DPOC se torna mais comum. ${ }^{2}$

Referente à idade e IMC, a média entre os grupos mantiveram-se próximas. No GC, a maioria dos indivíduos se apresentaram eutróficos, enquanto no GBEF observou-se predominância de indivíduos obesos. Ressalta-se que, de acordo com a literatura, a eutrofia dos portadores de DPOC pode derivar-se da quantidade elevada de massa gorda, fato esse que mascara uma possível desnutrição ou perda de massa magra. ${ }^{10} \mathrm{~A}$ desnutrição na DPOC está ligada a um esquema multifatorial, desde a ingestão inadequada de alimentos e o gasto energético aumentado. O gasto energético acrescido em portadores da doença pode ser atribuído ao hipermetabolismo, decorrente do aumento do trabaIho dos músculos respiratórios que induzem uma maior necessidade de oxigênio pelos mesmos. ${ }^{11}$

Por outro lado, portadores de DPOC com excesso de peso ou obesidade apresentam um efeito de proteção contra a mortalidade proveniente da doença, sendo que a justificativa em relação a isto é pouco esclarecida até o momento. O que se tem como evidência é a relação de risco entre o baixo IMC com a baixa sobrevida nos portadores de DPOC, decorrentes de varias razões, tais como a fraqueza muscular diafragmática, diminuição da função pulmonar e inflamação sistêmica. ${ }^{12}$

$\mathrm{Na}$ tabela 2 estão os achados obtidos na prova de função pulmonar dos participantes deste estudo. Nenhuma diferença significativa foi observada para as variáveis de função pulmonar entre os grupos estudados. Entretanto, uma observação importante deve ser ressaltada para o estadiamento da DPOC, pois não foi encontrado nenhum caso de estadiamento III (Severo) no GBEF, sendo que nos dois grupos estudados a maior parte dos pacientes encontrava-se em estadiamento IV, ou seja, na fase muito severa da doença.

A severidade da DPOC é dada pelo $\operatorname{VEF}_{1}$, que quanto menor o seu resultado no exame espirométrico, maior será o grau de obstrução das vias aéreas A maior parte da amostra deste estudo se mantém no último grau de severidade da doença (Grau IV, muito severo), o qual é qualificado por uma grave limitação do fluxo aéreo. $^{2}$

Pertinente aos resultados do pico de fluxo expiratório após a aplicação da BEF, os mesmos estão descritos na (Tabela 3) e nenhuma diferença estatisticamente significativa foi encontrada entre os grupos GC e GBEF, bem como dentro dos grupos.

A análise do delta de variação entre os testes de pico de fluxo expiratório revelou que no grupo tratado (GBEF) logo após a aplicação da BEF ( $\triangle$ PF1-PF2), houve uma melhora no pico de fluxo expiratório e esta decai após na medida de pico de fluxo final PF3 ( $\triangle$ PF2-PF3). Em contraste, o GC, em seu saldo final, obteve uma melhora entre PF1 e PF3.

No que diz respeito à $B E F$, além de não conter quaisquer substâncias químicas ou medicinais, elas são extensíveis longitudinalmente até cerca de 140\%, do seu comprimento original. ${ }^{13}$ Neste estudo, a BEF foi aplicada com uma tensão $>$ que $45 \%$ do seu tamanho original $^{8}$, porém não foi possível verificar uma diferença significativa da aplicação da BEF sobre os músculos que auxiliam na expiração (reto do abdome e oblíquo externo do abdome). A propriedade fisiológica da BEF engloba quatro funções, dentre as quais se destaca o efeito de suporte muscular, tendo a capacidade de dar suporte ao músculo aplicado ou grupamento muscular, durante um movimento ou atividade motora. Assim, possibilitaria a melhora da contração muscular de um músculo enfraquecido ou inibido e diminuiria a fadiga muscular. ${ }^{13}$

Tabela 2 - Caracterização da função pulmonar dos portadores de DPOC.

\begin{tabular}{lccc}
\hline Variáveis & $\begin{array}{c}\text { Grupo GC } \\
\text { Total }=\mathbf{6}\end{array}$ & $\begin{array}{c}\text { Grupo GBEF } \\
\text { Total = 11 }\end{array}$ \\
\hline CVF (\% predito) & $65,00 \pm 17,447$ & $54,45 \pm 12,597$ & 0,40 \\
VEF1 (\% predito) & $37,17 \pm 20,875$ & $31,73 \pm 15,704$ \\
VEF1/CVF (\% predito) & $47,17 \pm 14,303$ & $57,55 \pm 20,622$ \\
Estadiamento & & $3(27,3)$ \\
II, n (\%) & $1(16,7)$ & $--\boldsymbol{p}^{*}$ & -18 \\
III, n (\%) & $2(33,3)$ & $8(72,7)$ \\
IV, n (\%) & $3(50,0)$ & - \\
\hline
\end{tabular}

CVF: capacidade vital forçada; VEF1: volume expirado no primeiro segundo; VEF25-75\%: volume expirado forçado entre $25 \%$ e $75 \%$ da CVF; \# conforme a Global Initiative for Chronic Lung Disease (2014). Dados expressos em distribuição de frequência, média e desvio padrão; *valor de significância de p. Mann-Whiteny Test. 
Tabela 3 - Variáveis relativas ao Teste de Pico de Fluxo Expiratório nos três momentos da pesquisa.

\begin{tabular}{cccc}
\hline Variáveis & $\begin{array}{c}\text { Grupo GC } \\
\text { Total }=\mathbf{6}\end{array}$ & $\begin{array}{c}\text { Grupo GBEF } \\
\text { Total }=\mathbf{1 1}\end{array}$ & Valor de $\boldsymbol{p}^{*}$ \\
\hline PF1 (\% predito) & $57,31 \pm 22,51$ & $48,83 \pm 21,57$ & 0,66 \\
PF2 (\% predito) & ------ & $54,12 \pm 24,38$ & - \\
PF3 (\%predito) & $61,97 \pm 22,79$ & $47,60 \pm 16,53$ & 0,25 \\
$\Delta$ PF1-PF2 & ----- & 5,29 & - \\
D PF2-PF3 & ---- & $-6,52$ & - \\
$\Delta$ PF1-PF3 & 4,66 & $-1,22$ & 0,36 \\
\hline
\end{tabular}

PF1: primeiro encontro; PF2: segundo encontro; PF3: terceiro encontro; $\Delta$ : delta de variação. * valor de significância de p.Mann-Whiteny Test.

Tabela 4 - Características clínicas dos portadores de DPOC estratificados conforme resposta positiva e negativa ao uso da BEF nos três primeiros dias.

\begin{tabular}{lcc}
\hline Variáveis & $\begin{array}{c}\text { Grupo GBEF (delta negativo) } \\
\mathbf{n = 3}\end{array}$ & $\begin{array}{c}\text { Grupo GBEF (delta positivo) } \\
\mathbf{n}=\mathbf{5}\end{array}$ \\
\hline Sexo masculino, $\mathrm{n}(\%)^{\mathrm{b}}$ & $1(33 \%)$ & $3(60 \%)$ \\
Idade (anos) & $58,00 \pm 1,00$ & $57,50 \pm 6,58$ \\
IMC $\left(\mathrm{Kg} / \mathrm{m}^{2}\right)^{\mathrm{a}}$ & $23,67 \pm 5,68$ & $25,60 \pm 6,06$ \\
VEF1 & $23,33 \pm 3,78$ & $32,80 \pm 20,59$ \\
Classificação DPOC & & $2(40 \%)$ \\
GOLD II & $-\cdots---$ & $3(60 \%)$ \\
GOLD IV & $3(100 \%)$ & \\
\hline
\end{tabular}

IMC: Índice de massa corpórea; VEF1: volume expirado no primeiro segundo; GOLD: Global Initiative for Chronic Lung Disease 2014.

Tabela 5 - Variáveis da Escala de Dispneia nos diferentes momentos da análise do Pico de Fluxo Expiratório.

\begin{tabular}{|c|c|c|c|c|c|c|}
\hline \multirow[t]{2}{*}{ Variáveis } & \multicolumn{3}{|c|}{$\begin{array}{l}\text { Grupo GBEF (delta negativo) } \\
\qquad n=3\end{array}$} & \multicolumn{3}{|c|}{$\begin{array}{l}\text { Grupo GBEF (delta positivo) } \\
\qquad n=5\end{array}$} \\
\hline & PF1 & T. BEF & PF3 & PF1 & T. BEF & PF3 \\
\hline MRC1 & $1(33 \%)$ & & $1(33 \%)$ & & & \\
\hline MRC2 & $1(33 \%)$ & $1(33 \%)$ & $1(33 \%)$ & & $1(20 \%)$ & $1(20 \%)$ \\
\hline MRC3 & & & & $1(20 \%)$ & $2(40 \%)$ & \\
\hline MRC4 & & $1(33 \%)$ & & $3(60 \%)$ & $2(40 \%)$ & $4(80 \%)$ \\
\hline MRC5 & $1(33 \%)$ & $1(33 \%)$ & $1(33 \%)$ & $1(20 \%)$ & & \\
\hline
\end{tabular}

MRC: escala de dispneia Medical Research Council; MRC1: tenho falta de ar ao realizar exercício intenso; MRC2: tenho falta de ar quando apresso o meu passo, subo escadas ou ladeiras; MRC3: preciso parar algumas vezes quando ando no meu passo, ou ando mais devagar do que outras pessoas de minha idade; MRC4: preciso parar muitas vezes devido a falta de ar quando ando perto de $100 \mathrm{~m}$ ou poucos minutos de caminhada no plano; MRC5: sinto falta de ar que não saio de casa, ou preciso de ajuda para me vestir ou tomara banho sozinho; PF1: antes da aplicação da BEF; T. BEF = Tensionamento da Banda Elástica Funcional após 3 dias; PF3: dois dias após o tensionamento da BEF; (\%): porcentagem equivalente ao número de pacientes; (n): número de pacientes presentes em cada delta.

Apesar de não haver diferenças químicas ou físicas nas diferentes cores de BEF, a cor de um modo ou de outro pode influenciar aumentando ou diminuindo a tolerância quanto à pele, o que pode interferir nos resultados, embora não tenha até o momento nenhuma evidência científica que demonstre que a cor utilizada interfere no efeito desejado, fato este que é defendido pelos princípios da cromoterapia. ${ }^{14}$ A BEF vem sendo usada há mais de 20 anos, porém na maior parte destes estudos o foco se mantém na área ortopédica, apontando assim uma escassez de estudos em que a BEF é utilizada como auxílio na fisioterapia respiratória. ${ }^{15}$

Diante dos achados em nosso estudo, verificamos o perfil dos pacientes que responderam positivamente ao uso da BEF e os resultados estão descritos na (Tabela 4).

Os portadores de DPOC que responderam positivamente a BEF eram do sexo masculino, eutróficos, com uma menor obstrução das vias aéreas e com idade menos avançada. Apesar da melhor resposta ao uso da BEF na obstrução menos pronunciada das vias aéreas, $60 \%$ dos participantes com delta positivo se apresentaram no último estágio da doença (IV), demonstrando assim que a BEF beneficia estágios mais graves da DPOC também.

$\mathrm{Na}$ sequência, avaliamos a presença de dispneia nos diferentes momentos de análise do pico de fluxo expiratório e uso da BEF dos portadores de DPOC que responderam positivamente (Tabela 5).

Em nosso estudo, apesar de não encontrarmos diferença significativa do início para o fim do tratamento, observou-se uma melhora tanto do pico de fluxo expiratório quanto da dispneia (pelo MRC) 3 dias após a aplicação da BEF.

A dispneia, por sua vez, pode ser dessensibilizada por vários mecanismos e tratamentos, dentre eles destaca-se o medicamentoso, o treinamento físico 
(aeróbico e força muscular) e o treinamento muscular respiratório específico. ${ }^{3,16}$ Neste sentido, este estudo verificou que o uso da BEF pode influenciar positivamente na diminuição da dispneia dos portadores de DPOC que participam do programa de RP, sendo mais uma ferramenta de auxílio fisioterapêutico.

Para isto, algumas limitações devem ser superadas como a quantificação da distensão da banda elástica além do seu tamanho inicial, os processos alérgicos na pele que levam a perda de aderência da BEF e a determinação do tempo correto de uso das mesmas.

\section{CONSIDERACִÕES FINAIS}

A aplicação da BEF nos músculos expiratórios reto abdominal e oblíquo externo exerceu efeito positivo sobre o pico de fluxo expiratório dos portadores de DPOC com idade menos avançada, menor grau de obstrução de vias aéreas, eutróficos, em estadiamento avançado da doença e em programa de reabilitação pulmonar. Este efeito aconteceu imediatamente após a aplicação da BEF e teve repercussão positiva sobre a dispnéia destes sujeitos com diminuição desta sintomatologia.

Parece que a BEF, como complemento à fisioterapia respiratória, pode contribuir para a prática fisioterapêutica e assistência dos portadores de DPOC junto aos programas de RP. Sugerimos que novos estudos sejam desenvolvidos nesta área, levando em consideração a avaliação da força muscular respiratória e sua relação com o uso da BEF.

\section{REFERÊNCIAS}

1. Loivos LP. DPOC - Definições e Conceitos - As Bases Clínicas; Pulmão 2009; 1(1): 34-37.

2. Global Strategy For The Diagnosis, Management, And Prevention Of Chronic Obstructive Pulmonary Disease. GOLD/DPOC, 2014.

3. Camargo LACR, Pereira CAC. Dispneia em DPOC: além da escala modified Medical Research Council. Jornal Brasileiro de Pneumologia 2010; 36(5): 571-578. DOI: http://dx.doi.
org/10.1590/S1806-37132010000500008.

4. Fernandes AC, Bezerra OMPA. Terapia Nutricional na Doença Pulmonar Obstrutiva Crônica e suas Complicações Nutricionais. Jornal Brasileiro de Pneumologia 2006; 32(5): 461-71. DOI: http://dx.doi.org/10.1590/S1806-37132006000500014.

5. Hack LF. O uso de Bandagens Funcionais para Estimulação da Musculatura Respiratória de Pacientes Asmáticos. Monografia (Trabalho de Conclusão de Curso em Fisioterapia)Centro Universitário Feevale, Novo Hamburgo, 2009.

6. Leiner CG, et al. Expiratory peak flow rate. Standard values for normal subjects. Use a clinical test of ventilator function. The American Review of Respiratory 1963; 88: 644-51.

7. Moore KL, Dalley AF. Anatomia Orientada para Clínica. $5^{a}$ Edição. Brasil; 2011.

8. Junior NM. Therapy Taping Association: Método Therapy Taping- Conceito de Estimulação Tegumentar. São Paulo. 2009.

9. Pisoni TM. Prevalência da Doença Pulmonar Obstrutiva Crônica (DPOC) e Fatores de Risco Ambientais nos Bairros Mais Populosos de Novo Hamburgo [Tese]. Centro Universitário FEEVALE, Novo Hamburgo, 2007.

10. Cuppari L. Guias de Medicina Ambulatorial e Hospitalar. Nutrição Clínica no Adulto. UNIFESP, São Paulo: Manole. 2002.

11. Fernandes $A B S$. Reabilitação Respiratória em DPOC - A Importância da Abordagem Fisioterapêutica, Pulmão 2009; 1(1): 71-78

12. Cao C, Wang R, Wang J, Bunjhoo H, Xu Y, Xiong W (2012) Body Mass Index and Mortality in Chronic Obstructive Pulmonary Disease: A Meta-Analysis. PLoS ONE 2012; 7(8): e43892. DOI: http://dx.doi.org/10.1371/journal. pone.0043892.

13. Matos N. Kinesio Taping: Conceitos e Aplicações no Mundo do Desporto. Training. 2002.

14. Aguirre T. Kinesiology taping: Teoría y Práctica. Editora: Biocorp Europa S.L; 2010

15. Couto $E R$, et al. Bandagem elástica associada à fisioterapia respiratória convencional em pacientes com doença pulmonar obstrutiva crônica. Inspirar Movimento \& Saúde 2013; 26(5): 19-22.

16. Neto JECM, Amaral RO. Reabilitação Pulmonar e Qualidade de Vida Em Pacientes Com DPOC. Lato \& Sensu 2003; 4(1): 3-5. 